\title{
Retropharyngeal pleomorphic lipoma presenting as a neck mass- a rare case
}

\author{
J Phookan ${ }^{1}$, Dipjyoti Barman ${ }^{2}$, Shiv Kumar ${ }^{3}$, Sofia Okram ${ }^{4}$ \\ Pritam Chatterjee ${ }^{5}$, Priyam Sharma ${ }^{6}$, Daizy Brahma ${ }^{7}$ Jayanta Kr Das ${ }^{8}$ \\ I'Associate Proffessor, Department of ORL \& HNS, Gauhati Medical College \& Hospital, India) \\ ${ }^{2}$ (Registrar, Department of ORL \& HNS, Gauhati Medical College \& Hospital, India) \\ 3,4,5,6,7,8 (Junior Resident, Department of ORL \& HNS, Gauhati Medical College \& Hospital, India)
}

\begin{abstract}
Pleomormpic lipoma of the anterior neck is a rare entity. Although pleomorphic lipomas a benign tumour it may contain atypical cells. Histologically pleomorphic lipoma is composed of mature fat, bland spindle shaped mesenchymal cells and coarse "rope like" collagen bands In addition pleomorphic lopoma contains multi-neucleated florat like giant cells. It Enzinger \& Harvey ${ }^{[1]}$ in their original series of pleomorphic lipoma I spindle cell lipoma where there is little fat or no fat is a diagnostic challange.Immunohostochemical analysis by CD 34 positivity and desmin negativity is a diagnostic criteria. Here with we presenting a huge pleomorphic lipoma in the retropharyngeal space presenting as anterior neck swelling and sleep apnoea.
\end{abstract}

Keywords: Pleomorphic, retropharyngeal space, lipoma, benign neck swelling.

\section{Introduction}

Spindle cell lipoma $\backslash$ pleomorphic lipomma accounting for $1.5 \%$ of all adepocytic neoplasm, is a type of benign lipogenic tumour composed of primitive CD 34 positive spindle cells, floret like multineucleated giant cells and mature adepocytes.It was first described by Smookler BM ${ }^{[2]}$. It is mostly present in the posterior triangle of the neck., shoulder and back and other areas like oral cavity, genitals, parotid glands and extremeties.It is intradermal in many occasion. Retropharyngeal pleomorphic lipoma producing dysphagia and sleep apnoea is a very rare presentation. Here with we are presenting a patient of this nature for discussion.

\section{Case Report}

A 79 yrs old man presented to us with a neck mass extending from angle of the mandible to lower neck and with difficulty in deglutation and inability to breath in lying supine. The history was of a progressive swelling for $5 \backslash 6$ yrs. Clinical examination showed a soft compressible swelling occupying lt side of the neck extending from mandible to sternum and appears to be retro-sternal .On doing Indirect laryngoscopic examination the laryngeal inlet was found to be rotated towards $\mathrm{rt}$ and the posterior pharyngeal wall was found to be bulged from the level of the palate to the post cricoids area.MRI scan showed a hypoechoic lesion extending from the root of the neck upto the mandible and going retropharyngeal to the rt side displacing the great vessels to the lt. The lesion was seen extending from the skull base upto the superior mediastinum. FNAC showed the character of a lipoma.

Patient was planned for surgery. Tracheostomy was done as the patient could not be positioned for intubation. A horizontal incision was given from trapezius to midline on the $1 \mathrm{t}$ side of about $5 \mathrm{~cm}$. Anterior border of the sternocleidomastoid muscle was defined and incised to get the tumour and to have a good visual control of the great vessels. A Safe zone is defined in between the anterior margin of the sternocleidomastoid muscle superior belly of omohyoid muscle and superior thyroid pedicle to go into the other side of the neck down the inferior constrictor muscle without risking the middle thyroid vein, the recurrent laryngeal nerve, the thyroid vessels, the ansa hypoglossi. The whole pathlogy could be removed through this incision without going to the other of neck after releasing the thin fascial covering around it.

Biopsy showed typical "Floret" cells, which are large hyperchromatic multineucleated pleomorphic cells with lipoblast in intervening fibrous tissue in HE stain. Post operatively tracheostomy was closed after extubation. Post op period was uneventful. 


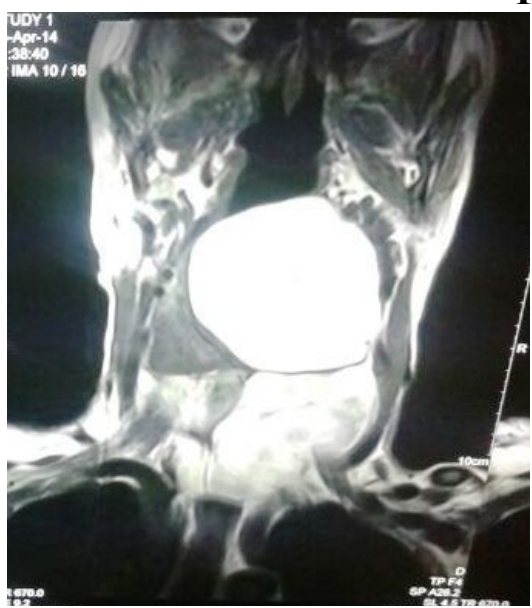

Fig 1. Preoperative MRI showing extentions of the lipoma
III. Figures

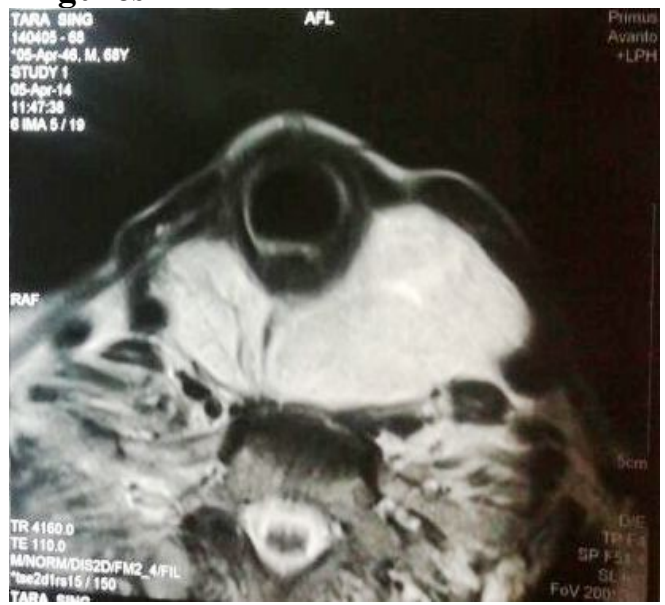

Fig 2. Preoperative MRI showing retropharyngeal extention of the lipoma

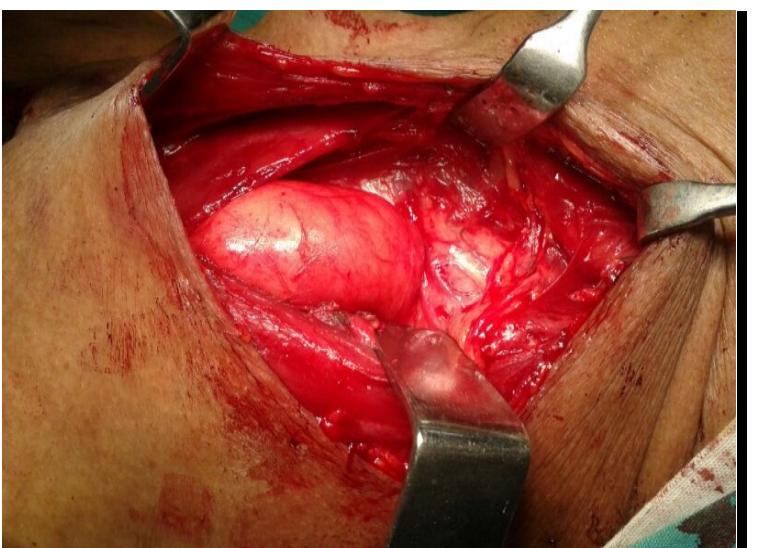

Fig 3. Intraoperative photograph showing the lipoma

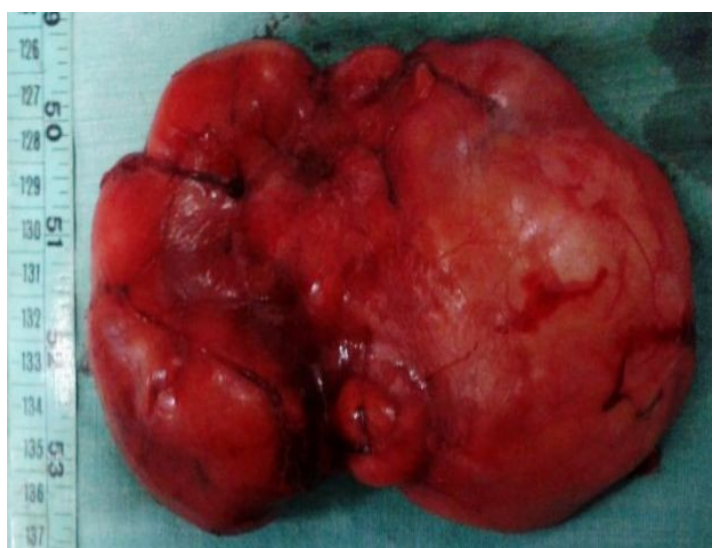

Fig 4. Post operative specimen
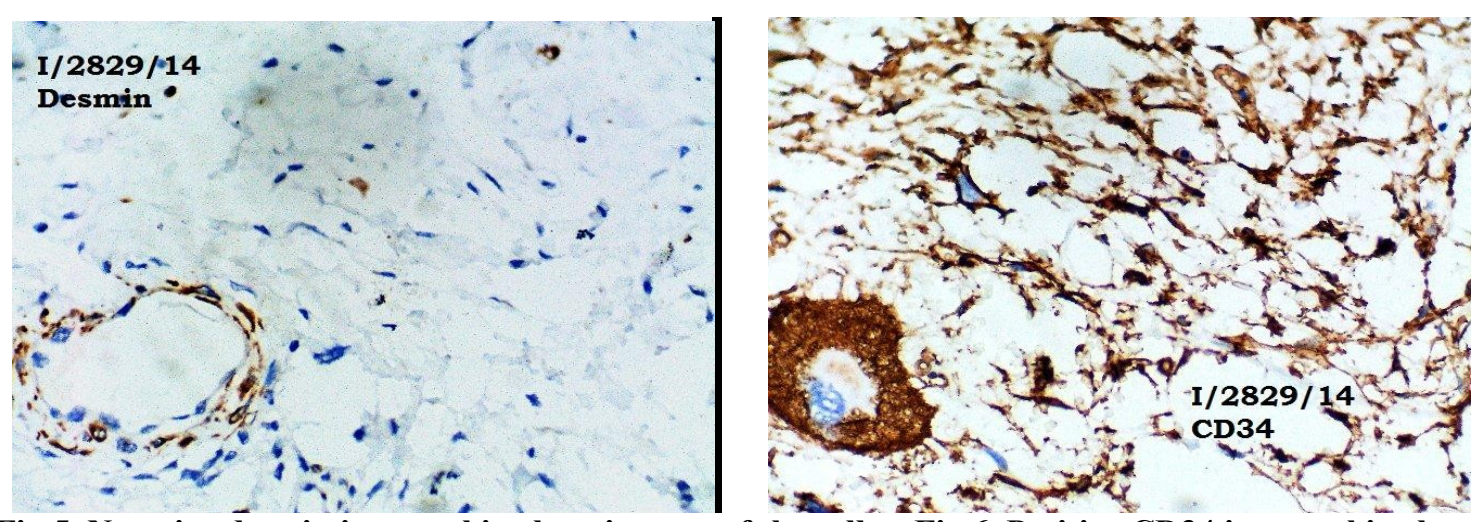

Fig 5. Negative desmin immunohistology in most of the cells Fig 6. Positive CD34 immunohistology

\section{Discussion}

Any retropharyhgeal swelling demands an extra attention as there is a possibility of air way obstruction apart from dysphagia. Taylor ${ }^{[3]}$ in 1877 first described retro pharyngeal lipoma. Retropharyngeal lipoma are rare tumours, only $10-13 \%$ lipoma occurs in head and neck ${ }^{[4]}$.Lipoma has got classification as Fibrolipoma, angiolipoma,infiltrating lipoma, spindle cell lipoma ${ }^{[5]}$.Pleomorphic lipomas are a rare variety of lipomas and constitute only $1.5 \%{ }^{[6]}$.In the head and neck areas it commonly occurs in the lateral neck and goes back to the shoulder and is common in $6^{\text {th }}$ and $7^{\text {th }}$ decade . Histologically " floret" pattern is characteristic.the most important issue is its differentiation from lipomasarcoma which can be done by estimating CD34 and Desmin. ${ }^{[7]}$. The retropharyngeal lipoma mostly present with dyspnoea and dysphagia.in our case sleep apnoea was the presenting symptom though there was a neck mass. This is most commonly found 
in elderly age group as it is slow growing tumour ${ }^{[8]}$.In our case ther was dysphonia also which was because of the rotation of the larynx.

Regarding the clinical examination the palpation finding is typical of a compressible swelling which is non tender. But on oral examination when the tumour extends from the skull base to the mediastinum, one can see the bulge in the posterior pharyngeal wall. On doing the laryngeal examination the rotation of the larynx was seen in our patient. CT scan and MRI scan both are help but mri gives a better delineation of the great vessels and the superior mediastinum. The dimension of the tumour dictates the size of the incision. We used a comparatively small incision of $5 \mathrm{~cm}$ from the trapezius to the mid line.We Define an area of safe zone in the neck to reach the contralateral neck. There is no such literature available regarding the safe zone.

\section{Conclusion}

Pleomorphic lipoma in the retropharyngeal space is a rare tumour. Proper radiological evaluation and cytological assessment to rule out sarcoma is a pre operative compulsion and saving all the vital structures with complete extirpation of the tumour is the key to the success.

\section{References}

[1]. Enzinger F.M, Harvey D A, Spindle cell lipoma description. Cancer. 1975 Nov;36(5):1852-9

[2]. Smookler B M ,Enzinger FM, Pleomorphic lipoma : A benign tumour simulating liposarcoma. Cancer.1981, 47,126 - 133.

[3]. Taylor F. Fatty tumour behind the pharynx, Trans Pathol Soc.london 1877;28:216-218

[4]. Hadad FS, Hadad FF, Zaytoun G, Retropharyngeal lipoma a benign yet potentially lethal condition. Case presentation and review of literature. Neurosurgery . 2005: 15: 145-154.

[5]. Rosai \& Ackermans, Surgical pathology. 2: 2275 -2277.

[6]. Sternbergs diagnostic surgical pathology, $4^{\text {th }}$ edition. 2004; 1: 155-157.

[7]. AzzopardiJG, Iocco J,Salm R, Pleomorphic lipoma: A tumour simulating liposarcoma. Histopathology.1983;7: 511-523.

[8]. Akhtar J, Shaykhon M,Crocker J, D’ Souza AR , Retrpharyngeal lipoma causing dysphagia. Eur Archive Otolaryngol 2001; 258:458-459. 